\title{
Open cell conducting foams for high synchrotron radiation accelerators
}

\author{
S. Petracca ${ }^{*}$ and A. Stabile \\ Dipartimento di Ingegneria, Universita' del Sannio, Palazzo Dell'Aquila Bosco Lucarelli, \\ Corso Garibaldi, 107 - 82100, Benevento, Italy
}

(Received 26 October 2012; revised manuscript received 3 June 2014; published 28 August 2014)

The possible use of open cell conductive foams in high synchrotron radiation particle accelerators is considered. Available materials and modeling tools are reviewed, potential pros and cons are discussed, and preliminary conclusions are drawn.

DOI: 10.1103/PhysRevSTAB.17.083503

PACS numbers: 29.20.db, 41.60.Ap, 88.40.fh

\section{INTRODUCTION}

Molecular gas desorption from the beam pipe wall due to synchrotron radiation should be properly taken into account in the design of high-energy particle accelerators and storage rings. This has been a major (solved) challenge for the Large Hadron Collider, will be even more critical for the HE-LHC, in view of its higher level of synchrotron radiation [1], and will be a crucial issue for the successful operation of the proposed electron-positron Higgs factories [2].

In the CERN Large Hadron Collider [3] a copper-coated stainless steel beam screen (the liner) is kept at $\approx 20 \mathrm{~K}$ by active Helium cooling, and effectively handles the heat load represented by synchrotron radiation, photoelectrons, and image-charge losses. A large number $\left(\sim 10^{2} \mathrm{~m}^{-1}\right)$ of tiny slots are drilled in the beam screen wall (see Fig. 1) in order to maintain the desorbed gas densities below a critical level (e.g., $\sim 10^{15}$ molecules $/ \mathrm{m}^{3}$ for $H_{2}$ ) by allowing desorbed gas to be continuously cryopumped toward the stainless steel cold bore (coaxial to the beam screen) of the superconducting magnets, which is kept at $1.9 \mathrm{~K}$ by superfluid helium. Above such critical density levels, nuclear scattering in the residual gas would limit the beam luminosity lifetime, eventually originate high-energy protons which may cause thermal runaway, and ultimately cause quenching of the superconducting magnets.

The size, geometry, placement, and density of the pumping slots affect the beam dynamics and stability in a way that is synthetically described by the longitudinal and transverse beam coupling impedances [4]. The slot geometry and placement should be further chosen so as to minimize the effect of trapped (cutoff) modes, and to prevent the possible coherent buildup of radiation in the

\footnotetext{
petracca@sa.infn.it

arturo.stabile@sa.infn.it
}

Published by the American Physical Society under the terms of the Creative Commons Attribution 3.0 License. Further distribution of this work must maintain attribution to the author $(s)$ and the published article's title, journal citation, and DOI.
TEM waveguide limited by (the outer surface of) the pipe and the cold bore [5].

Open cell conducting foams could be an interesting candidate for materials to help fulfill the above general requirements in beam screen design. In addition, the surface roughness of conducting foams may help reduce the effective secondary-emission yield (SEY) [6], thus alleviating the electron cloud buildup phenomenon [7] and related instabilities [8]. In this paper we present a brief review of open cell conducting foams' properties and modeling tools and discuss at a very preliminary level the pros and cons of their possible use in high synchrotron radiation accelerators.

\section{OPEN CELL METAL FOAMS}

Open cell metal foams (OCMF) can be produced by vapor (or electro) deposition of metal on an open cell polymer template, followed by polymer burn off, and a final sintering step to densify the ligaments. Alternatively, they can be synthetized by infiltration and casting of molten metal into a solid mold, consisting of packed (nonpermeable) templates of the foam pores, followed by burnout and removal of the mold [9]. Both processes result in highly gas-permeable reticular materials, where only a $3 \mathrm{D}$ web of thin conducting ligaments survives. The typical structure of OCMFs is displayed in Fig. 2. The key structural parameters of OCMF are the "pore" size and the porosity (volume fraction of pores). Pore sizes in the range from $10^{-4}$ to $10^{-3} \mathrm{~m}$ and porosities in the range 0.7-0.99 are typical. These two parameters determine the gas permeability of the material, and, together with the electrical properties of the metal matrix, its electrical characteristics. OCMF have remarkable structural properties [low density and weight, high (tensile and shear) strength-to-weight ratio, nearly isotropic load response, low coefficient of thermal expansion], as summarized in Table I, which qualified them among the most interesting new materials for aerospace applications. Aluminum and copper OCMF are presently available off the shelf from several manufacturers worldwide, and are relatively inexpensive. They can be coated, with silver, titanium, or platinum, for special purpose 


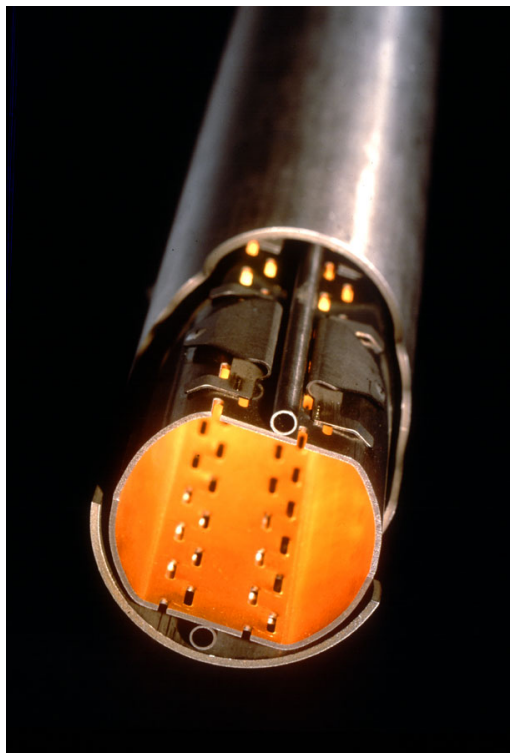

FIG. 1. The LHC slotted copper-plated beam screen and stainless steel cold bore.

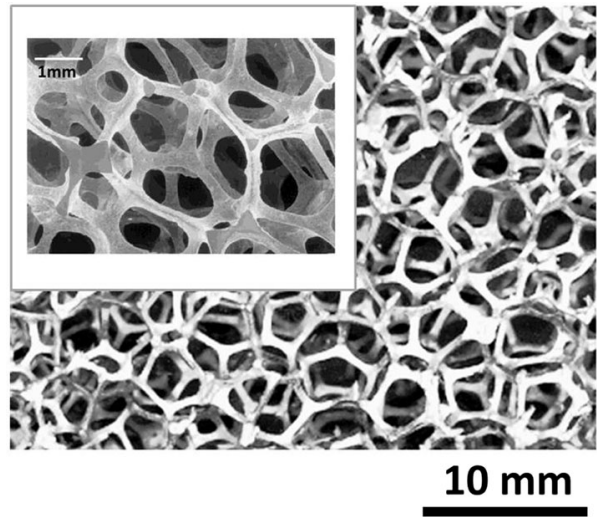

FIG. 2. A typical open cell metal foam, at two different viewing scales.

applications. Steel and brass foams, as well as silver, nickel, cobalt, rhodium, titanium, or beryllium foams have been also produced.

The Weaire-Phelan (WP) space-filling honeycombs are credited to provide the natural (i.e., Plateau's minimal surface principle compliant) model of OCMF with equally sized (but possibly unequally shaped) pores [11]. The WP unit cell consists of a certain arrangement of (irregular) polyhedra, namely, two pentagonal-face dodecahedra (with tetrahedral symmetry $T_{h}$ ), and six tetrakaidecahedra (with antiprysmatic symmetry $D_{2 d}$ ) featuring two hexagonal and twelve pentagonal faces [11]. A computer generated WP honeycomb is displayed in Fig. 3, and its visual similarity to Fig. 2 is apparent.

A powerful open-source cross-platform software project for 3D morphological characterization and
TABLE I. Structural properties of $\mathrm{Al}$ and $\mathrm{Cu}$ open cell metal foams from [10].

\begin{tabular}{lccc}
\hline \hline & Units & $\mathrm{Al}$ & $\mathrm{Cu}$ \\
\hline Compressive strength & {$[\mathrm{MPa}]$} & 2.5 & 0.9 \\
Tensile strength & {$[\mathrm{MPa}]$} & 1.2 & 6.9 \\
Shear strength & {$[\mathrm{MPa}]$} & 1.3 & 1.3 \\
Elastic modulus (compressive) & {$[\mathrm{MPa}]$} & $1 . \times 10^{2}$ & $7.3 \times 10^{2}$ \\
Elastic modulus (tensile) & {$[\mathrm{MPa}]$} & $1 . \times 10^{2}$ & $1 . \times 10^{2}$ \\
Shear modulus & {$[\mathrm{MPa}]$} & $2 . \times 10^{2}$ & $2.8 \times 10^{2}$ \\
Specific heat & {$\left[\mathrm{J} / \mathrm{g}^{0} \mathrm{C}\right]$} & 0.89 & 0.38 \\
Bulk thermal condition & {$\left[\mathrm{W} / \mathrm{m}^{0} \mathrm{C}\right]$} & 5.8 & 10.1 \\
Thermal expansion & {$\left[1 /{ }^{0} \mathrm{C}\right]$} & $2.4 \times 10^{-5}$ & $1.7 \times 10^{-5}$ \\
$\quad$ coefficient. & {$[\Omega / \mathrm{m}]$} & $7.2 \times 10^{-7}$ & $6.5 \times 10^{-7}$ \\
Bulk resistivity & {$\left[{ }^{0} \mathrm{C}\right]$} & 660 & 1100 \\
Melting point & \multicolumn{3}{c}{} \\
\hline \hline
\end{tabular}

modeling of cellular materials, including OCMF, is under development [12].

\section{A. Electrical properties of conducting foams}

Electromagnetic modeling of OCMFs has been thoroughly investigated during the last decade. A numerical approach based on Weiland finite integration technique (FIT, [13]) has been proposed by Zhang et al. [14] to compute the (frequency, thickness, and angle of incidencedependent) reflection coefficient of $\mathrm{SiC}$ foam and optimize
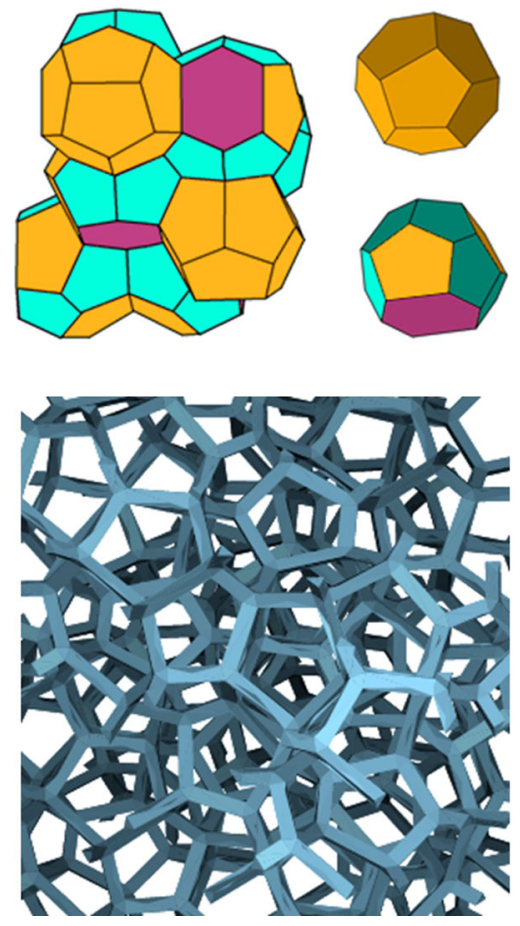

FIG. 3. The Wearie-Phelan honeycomb cell (top left), its constituent polyhedra (top right), and a numerically simulated reticulated foam thereof (bottom). 
its design. The main limitation of Zhang's analysis is in the use of a simple body-centered cubic unit cell foam model, for easiest numerical implementation. The FIT scheme, however, may accommodate more complicated and realistic foam-cell geometries, including in principle the WP one.

In the quasistatic limit $\lambda \longrightarrow 0$, the conductivity of OCMFs can be computed using effective medium theory (EMT), for which several formulations exist (see, e.g., [15], [16] for a review). These include (i) the self-consistent approach [17], credited to Bruggemann, where inclusions are thought of as being embedded in the (actual) effective medium; (ii) the differential scheme, whereby inhomogeneities are incrementally added to the composite, ${ }^{1}$ until the final concentration is reached, so that at each step the inclusions do not interact and do not modify the field computed at the previous step [18]; and (iii) the effectivefield methods, whereby interaction among the inclusions is described in terms of an effective field acting on each inclusion, accounting for the presence of the others. Two main versions of this method exist, credited to Mori-Tanaka [19] and Levin-Kanaun [20], differing in the way the effective field is computed (average over the matrix only, or average over the matrix and the inclusions, respectively).

The self-consistent approach yields

$$
\sigma_{\text {eff }}=\sigma_{0}(1-p \psi),
$$

where $\sigma_{0}$ is the bulk metal conductivity, $p$ is the porosity (volume fraction of the vacuum bubbles), and $\psi$ is a morphology-dependent factor. The differential approach yields

$$
\sigma_{\text {eff }}=\sigma_{0}(1-p)^{\psi},
$$

while the Mori-Tanaka/Levin-Kanaun approaches yield

$$
\sigma_{\mathrm{eff}}=\sigma_{0} /\left(1+\frac{\psi p}{1-p}\right)
$$

All equations (1)-(3) merge, as expected, in the $p \rightarrow 0$ limit. The above models are synthetically compared to measured values of the static conductivity in Fig. 4, for aluminum foams. For aluminum foams, all of these models predict larger conductivity than observed in measurements. ${ }^{2}$ This has been attributed to significant oxide formation on the solid web [21]. Equation (2) agrees in form with predictions based on percolation theory

\footnotetext{
${ }^{1}$ In this approach, the total concentration of inhomogeneities does not coincide with the volume fraction $p$, because at each step new inclusions may be placed where old inclusions have already been set.

${ }^{2}$ It should be noted that open and closed cell metal foams behave similarly in terms of electrical conductivity, while being markedly different in regards to thermal conductivity, due to the different role of convective flow.
}

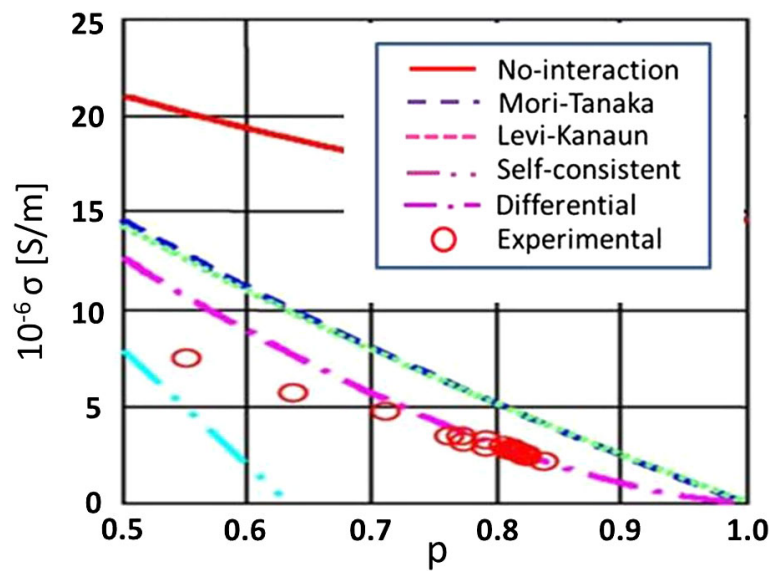

FIG. 4. Static conductivity vs porosity for aluminum based foams from different EMT models. Measured values are also shown (adapted from [23]).

[22]—although strictly speaking there is no threshold here beyond which the conducting component disconnects.

Measurements of the microwave electromagnetic shielding efficiency of OCMF panels [24] indicate that a simple Drude model

$$
\sigma(\omega)=\frac{\omega_{p}^{2} \epsilon_{0}}{l \omega+\nu}
$$

provides a good description of the frequency-dependent conductance of metallic foams. Typically, the relevant plasma and collision frequencies, $\omega_{p}$ and $\nu$, are of the order of a few tens of $\mathrm{GHz}$ and a few tens of $\mathrm{KHz}$, respectively. These are notably much smaller ${ }^{3}$ than their solid metal counterparts (typically in the $\mathrm{PHz}$ and $\mathrm{GHz}$ ranges, respectively [26]).

${ }^{3}$ In the light of the well-known formula

$$
\omega_{p}^{2}=\frac{N_{e} q^{2}}{m_{e} \epsilon_{0}},
$$

this can be explained as due to a reduction of the electron density $N_{e}$, roughly by a factor $(1-p), p$ being the porosity and a parallel (large) increase of the effective electron mass. Pendry et al. have shown that the effective mass of an electron in a thin-conducting-wire lattice or web is essentially set by its electromagnetic momentum, yielding [25]

$$
\omega_{p}^{2} \sim \frac{c^{2}}{D^{2} \log (D / d)},
$$

where $d$ and $D$ are the typical conducting-ligament and pore diameters, respectively, and

$$
\nu \sim \frac{\epsilon_{0} \omega_{p}^{2}}{\pi \sigma_{0}}\left(\frac{D}{d}\right)^{2} .
$$




\section{OCMF impedance and skin depth}

Throughout a typical beam current frequency spectrum, OCMF and bulk metals behave quite differently. ${ }^{4}$ In bulk metals, $\omega \ll \nu \ll \omega_{p}$ so that the characteristic impedance $Z_{m}$ and (complex) propagation constant $\tilde{k}_{m}$ can be written

$$
Z_{m} \sim \frac{1+\imath}{\sqrt{2}}\left(\frac{\omega \nu}{\omega_{p}^{2}}\right)^{1 / 2} Z_{0}, \quad \tilde{k}_{m} \sim \frac{1-\imath}{\sqrt{2}} k_{0}\left(\omega_{p}\right) \sqrt{\frac{\omega}{\nu}},
$$

$Z_{0}$ and $k_{0}(\omega)=\omega / c$ being the vacuum characteristic impedance and propagation constant, respectively. It is seen that in metallic conductors, both $Z_{m}$ and $\tilde{k}_{m}$ are $\propto \omega^{1 / 2}$.

In OCMFs, on the other hand, $\nu \ll \omega \ll \omega_{p}$ throughout the beam current spectrum, so that the material exhibits a plasmonic behavior. The OCMF wall (characteristic) impedance $Z_{f}$ and (complex) propagation constant $\tilde{k}_{f}$ are thus given (to lowest order in the small quantities $\nu / \omega$ and $\left.\omega / \omega_{p}\right)$ by

$$
Z_{f} \sim Z_{0}\left(\frac{\nu}{2 \omega_{p}}+\imath \frac{\omega}{\omega_{p}}\right), \quad \tilde{k}_{f} \sim k_{0}\left(\omega_{p}\right)\left(\frac{\nu}{2 \omega}-\imath\right) .
$$

Hence, the OCMF characteristic resistance $R_{f}=\operatorname{Re}\left[Z_{f}\right]$ and skin depth $\delta_{f}$ are both frequency independent, and, e.g., for the case of high-grade $\left(\rho \approx 5.5 \times 10^{-10} \Omega \mathrm{cm}^{-1}\right.$ at $20 \mathrm{~K}$ ) copper foam with $p=0.9$, both fairly small:

$$
R_{f} \sim \frac{Z_{0}}{2} \frac{\nu}{\omega_{p}} \approx 1.4 \times 10^{-5} \Omega, \quad \delta_{f} \sim \frac{c}{\omega_{p}} \approx 6 \times 10^{-4} \mathrm{~m} .
$$

The OCMF characteristic reactance

$$
X_{f}=\operatorname{Im}\left[Z_{f}\right] \sim \imath Z_{0} \frac{\omega}{\omega_{p}}
$$

on the other hand, is large compared to that of bulk metal, and grows linearly with $\omega$. For the case, e.g., of high-grade copper foam with $p=0.9, X_{f} \approx 0.5 \Omega$ at $10^{4} \mathrm{~Hz}$.

\section{B. Superconducting foams}

At the operating temperature of the LHC beam screen $(\sim 20 \mathrm{~K})$, both aluminum and copper exhibit a fairly large conductivity $\left(\sim 10^{7} \Omega^{-1} \mathrm{~cm}^{-1}\right)$, but neither of them is superconducting.

\footnotetext{
${ }^{4}$ The typical power spectrum of a bunched beam in a ring collider consists of lines at integer multiples of $f_{0}=c / \delta_{b}, \delta_{b}$ being the bunch spacing, with an envelope approximately $\propto \cos ^{2}$. In the LHC the $-20 \mathrm{~dB}$ bandwidth is $\sim 1 \mathrm{GHz}$, roughly $10^{5}$ times the circulation frequency $\omega_{R}$, and $10^{-1}$ times the cutoff frequency of the lowest waveguide mode of the beam screen [27].
}

Superconducting OCMFs have been discussed in [28]. Foamed ceramic superconductors [in particular, YBCOs (yttrium, barium, copper, oxide)], with critical temperature well above $20 \mathrm{~K}$ [high-temperature superconductors (HTS)] have also been already manufactured [29]. These materials may likely exhibit a very low SEY.

Besides being technologically appealing, HTS foams are conceptually interesting materials, where a percolating electric current coexists with a percolating magnetic flux [30]. A substantial body of experimental results on the electrical properties of thin-film HTS is available [31], and these are reasonably well accounted for [32] by a simple two-fluid Drude model [33]. However, the electrical properties of foamed HTS have been investigated so far, to the best of our knowledge mostly at very low frequencies [34].

\section{METAL FOAM vs PERFORATED METAL PATCHED PIPES}

In this section we shall attempt to draw a comparison between beam screens using perforated metal patches for outgassing vs pipes using OCMF patches, in terms of the relevant vacuum and beam coupling impedance features.

\section{A. Vacuum issues}

The vacuum dynamics for each molecular species that may be desorbed from the wall by synchrotron radiation can be described by the following set of (coupled) rate equations [35]:

$$
\left\{\begin{array}{l}
V \frac{d n}{d t}=q-a n+b \Theta \\
F \frac{d \Theta}{d t}=c n-b \Theta
\end{array} .\right.
$$

Here $n\left[m^{-3}\right]$ and $\Theta\left[m^{-2}\right]$ are the volume and surface densities of desorbed particles, respectively, and $V$ and $F$ represent the volume and wall area of the beam screen per unit length, respectively.

The first term on the right-hand side of the first rate equation represents the number of molecules desorbed by synchrotron radiation per unit length and time, and is given by

$$
q=\eta \dot{\Gamma}
$$

where $\eta$ is the desorption yield (number of desorbed molecules per incident photon) and $\dot{\Gamma}$ is the specific photon flux (number of photons hitting the wall per unit length and time).

The second term represents the number of molecules that are removed per unit time and unit length by either sticking to the wall or escaping through the pumping holes and slots. The $a$ coefficient in (9) can accordingly be written

$$
a=\frac{\langle v\rangle}{4}(s+f) F,
$$


TABLE II. Typical values of the parameters in (14) from [35].

\begin{tabular}{lcc}
\hline \hline$V$ & liner volume (per unit length) & $1.3 \times 10^{-3} \mathrm{~m}^{3} / \mathrm{m}$ \\
$F$ & liner surface (per unit length) & $0.14 \mathrm{~m}^{2} / \mathrm{m}$ \\
$\eta$ & desorption yield & $5 \times 10^{-4}$ \\
$\Gamma$ & photon flux (200 mA beam) & $3.14 \times 10^{16} \mathrm{~s}^{-1} \mathrm{~m}^{-1}$ \\
$s$ & sticking probability & 0.6 \\
$\kappa$ & recycling coefficient & $5 . \times 10^{-21} \mathrm{~m}^{2}$ \\
$\nu_{0}$ & vibrational frequency & $10^{13} \mathrm{~s}^{-1}$ \\
$W$ & activation energy & $0.035 \mathrm{eV} / \mathrm{molecule}$ \\
\hline \hline
\end{tabular}

where $\langle v\rangle \approx(8 k T / \pi m)^{1 / 2}$ is the average molecular speed, $m$ being the molecular mass, $k$ the Boltzmann constant and $T$ the absolute temperature, $\langle v\rangle / 4$ is the average number of collisions of a single molecule per unit time and unit wall surface, $s$ is the sticking probability, and $f$ is the escape probability. The third term accounts for thermal or radiation induced recycling of molecules sticking at the walls. The $b$ coefficient in (9) can accordingly be written

$$
b=\kappa \dot{\Gamma}+F \nu_{o} \exp (-W / k T)
$$

Here the first term accounts for radiation induced recycling, described by the coefficient $\kappa\left[\mathrm{m}^{2}\right]$, while the second term describes thermally activated recycling, $\nu_{0}$ being a typical molecular vibrational frequency and $W$ a typical activation energy.

The $b \Theta$ term appears with reversed sign on the righthand side of the second rate equation, where it represents the number of molecules desticking from the wall surface per unit time and unit length. The first term on the righthand side of this equation represents the number of molecules sticking to the wall, per unit time and unit length, when [compare with Eq. (11)]

$$
c=\frac{\langle v\rangle}{4} s F .
$$

At equilibrium, $\dot{n}=\dot{\Theta}=0$, and the rate equations yield

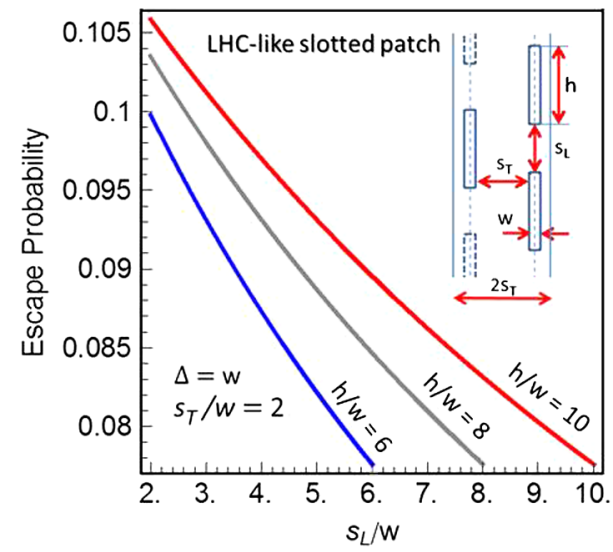

$$
\left\{\begin{array}{l}
n_{\mathrm{eq}}=\frac{4 \eta \dot{\Gamma}}{\langle v\rangle f F} \\
\Theta_{\mathrm{eq}}=\frac{s}{f} \frac{\eta \dot{\Gamma}}{\kappa \dot{\Gamma}+F \nu_{o} \exp (-W / k T)}
\end{array} .\right.
$$

Typical values (for LHC) of the parameters in (14) are collected in Table II [35]. The equilibrium molecular densities in (14) should not exceed some critical values for safe operation [35].

\section{Beam screen with perforated metal patches}

For a beam screen wall with vanishing thickness the molecular escape probability $f$ in (11) and (14) will be given by the holey fraction $\xi_{h}$ of the total wall surface. The desorption yield $\eta$ and the sticking probability $s$ will likewise differ from those of solid metal by a factor $\left(1-\xi_{h}\right)$.

For a thick perforated wall, the escape probability will be $f=\chi \xi_{h}$ where the factor $\chi<1$ (named after Clausing), takes into account the possibility that molecules may stick at the hole internal surface rather than escaping outside [36].

If only a fraction $\xi_{p}$ of the beam screen surface is covered by perforated patches, the beam screen escape probability will be $f=\xi_{p} \chi \xi_{h}$.

In the LHC the pumping holes are thin slots parallel to the pipe axis [37]. The slots are confined to four narrow ( $\approx 9 \mathrm{~mm}$ wide) strip-shaped patches, running parallel to the pipe axis, as seen in Fig. 1. The escape probability of an LHC-like slotted patch (strip) is displayed in the left panel of Fig. 5. Stiffness requirements set a lower limit to the axial $\left(s_{L}\right)$ and transverse $\left(s_{T}\right)$ slot spacings at roughly twice the slot width $w$. In Fig. 5 we assume $s_{T}=2 w$ and plot the escape probability vs the scaled longitudinal slot separation $s_{L} / 2 w$, for three typical values of the slots height-to-width ratio $h / w$. The wall thickness $\Delta$ is assumed as equal to $w$, yielding a Clausing factor $\chi \approx 0.68$ [38].

In the LHC the slotted strips represent roughly one-half of the beam screen surface. The slots are $\approx 8 \mathrm{~mm}$ long, on

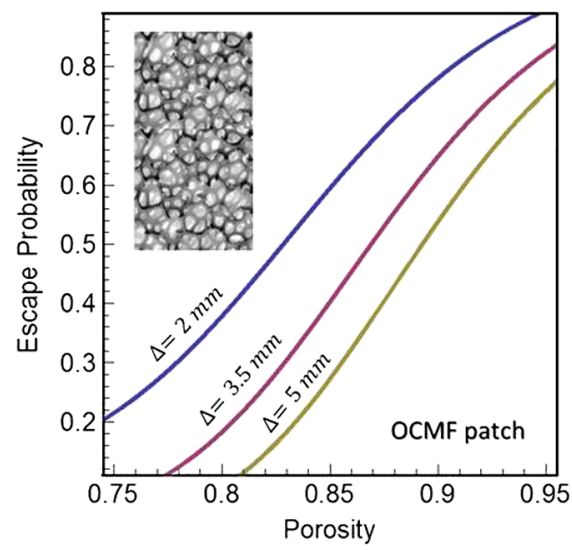

FIG. 5. Left: escape probabilities for LHC-like slotted patches. Right: escape probability for OCMF patches. 
average, and $\approx 1.5 \mathrm{~mm}$ wide, with $s_{L} \approx s_{T} \approx 3 \mathrm{~mm}$. The holey fraction of the total beam screen surface is roughly $5 \%$, and the escape probability is $\approx 3.5 \%$.

\section{Beam screen with OCMF patches}

Gas permeability of OCMFs in the molecular flow regime is described by Knudsen diffusivity [36]. The dependence of this latter on foam porosity was fitted numerically in [39] and [40], using computer simulations of molecular diffusion based on tomographic data of actual OCMF specimens. An exponential blowup of Knudsen diffusivity $D_{K}$ was observed, above some porosity threshold, possibly due to the creation of long-range molecular pathways.

From Knudsen diffusivity the escape probability of OCMF patches can be readily obtained, using Fick's (diffusion) law, and is displayed in Fig. 5 (right panel) as a function of porosity, for different values of the OCMF wall thickness $\Delta$.

It is seen from Fig. 5 that typical OCMF patches, e.g., with $p=0.9$, feature fairly larger escape probabilities, compared to slotted patches.

OCMF walls are also expected to provide better EM shielding compared to slotted ones. Hence, gas molecules sticking inside the foam pores will be less exposed to recycling, resulting in possibly smaller values of the recycling factor $\kappa$ in (12) and (14).

\section{B. Beam coupling impedance and parasitic losses}

Beam coupling impedances provide a synthetic description of the electromagnetic beam pipe interaction. Here, for the sake of brevity, we shall confine our discussion to the longitudinal impedance. The absolute value and the imaginary part of the latter are inversely proportional to the threshold currents for (single-bunch) microwave instability and Landau damping suppression, respectively, and hence quite relevant to beam stability [4], while the real part determines the parasitic loss (energy lost by the beam per unit pipe length), via [4]

$$
\Delta \mathcal{E}=\frac{1}{2 \pi} \int_{-\infty}^{+\infty}|I(\omega)|^{2} \operatorname{Re}\left[\bar{Z}^{\|}(\omega)\right] d \omega,
$$

where $I(\omega)$ and $Z^{\|}(\omega)$ are the beam current spectrum and longitudinal impedance per unit length, respectively.

The following relationship exists (to first order in the wall impedance) between the longitudinal impedance per unit length $\bar{Z}_{\|}$of a patched-wall beam screen and the (known) longitudinal impedance per unit length $\bar{Z}_{\|}^{(0)}$ of the same pipe with a perfectly conducting wall [41]

$$
\bar{Z}_{\|}=\bar{Z}_{\|}^{(0)}+\frac{\epsilon_{0} Y_{0}}{c \Lambda^{2}} \oint_{\partial S} Z_{w}(s)\left|E_{0 n}(s)\right|^{2} d s,
$$

where $Z_{w}$ is the (local) Leontóvich impedance [42] of the patched wall, $\partial S$ is the pipe cross-section contour, $Y_{0}=$ $\left(\epsilon_{0} / \mu_{0}\right)^{1 / 2}$ the vacuum admittance, $\Lambda$ the beam linear charge density, and $E_{n}^{(0)}$ the (known) field component normal to the pipe wall in the perfectly conducting pipe. ${ }^{5}$ According to (16), perforated or slotted or OCMF patches placed where the normal field component $E_{n}^{(0)}$ is minimal will have minimum impact on the longitudinal impedance. Hence, a circular beam screen, featuring a uniform field along $\partial S$, represents the worst case, where the longitudinal (and transverse) beam coupling impedances are simply proportional to the average of the wall impedance, assumed as piecewise constant

$$
\left\langle Z_{\mathrm{wall}}\right\rangle=\sum_{i} \xi_{i} Z_{\mathrm{wall}}^{(i)},
$$

$\xi_{i}$ being the surface fraction covered by patches with wall impedance $Z_{\text {wall }}^{(i)}$.

\section{Impedance of perforated patch}

The effective wall impedance of a perforated patch can be computed under the Bethe approximation where the holes and slots are (much) smaller than the shortest wavelength in the beam spectrum, yielding [43], [44]

$$
Z_{s}^{(0)}=\imath Z_{0}\left(\frac{\omega}{c}\right) \alpha_{\mathrm{tot}}^{(i)} n_{\sigma}
$$

$Z_{0}=\left(\mu_{0} / \epsilon_{0}\right)^{1 / 2}$ being the vacuum characteristic impedance, $\alpha_{\text {tot }}^{(i)}$ the total internal polarizability of the holes and slots, and $n_{\sigma}$ their surface density.

In [45] the more general case, relevant for the LHC, of a circular perforated beam screen with inner radius $a$ and thickness $\Delta$ surrounded by a circular coaxial lossy tube (the cold bore) with radius $b$ was investigated.

It was found that the beam screen wall impedance can still be cast in the form of Eq. (18), after the formal substitution

$$
\alpha^{(i)} \longrightarrow \alpha^{(i)}+F \alpha^{(e)},
$$

where the superfix $(e)$ identifies the total external polarizability of the holes and slots in a wall with thickness $\Delta$, and [44], [45]

$$
F=-\frac{\alpha^{(e)}}{\alpha^{(i)}}\left[1+\imath\left(1+\frac{b}{a+\Delta}\right) \frac{Z_{\mathrm{cb}}}{Z_{h}^{(0)}}\right]^{-1},
$$

$Z_{\mathrm{cb}}$ being the cold bore wall impedance. ${ }^{6}$ The wall reactance is negligibly affected by the presence of the

\footnotetext{
${ }^{5} \mathrm{~A}$ similar formula exists for the (dyadic) transverse impedance [41].

${ }^{6}$ In this formula $Z_{\mathrm{cb}}$ is the impedance of both the the cold bore and outer beam screen walls.
} 
TABLE III. Summary of Leontóvich impedances. High-grade copper with $\rho \approx 5.5 \times 10^{-10} \Omega \mathrm{cm}^{-1}$ at $20 \mathrm{~K}$. OCMF with $1 \mathrm{~mm}$ pores and $0.1 \mathrm{~mm}$ ligaments. Slots in a perfectly conducting wall as in Fig. 5, with $w=\Delta=1.5 \mathrm{~mm}, h=10 \mathrm{w}$, and $s_{T}=s_{L}=2 w$.

\begin{tabular}{llccc}
\hline \hline & Solid copper patch & OCMF patch & OCMF roughness & Slotted patch (perfect conductor) \\
\hline$\left(\omega_{R} / \omega\right) R_{\text {wall }},[\Omega]$ & $4.9 \times 10^{-6} \sqrt{\omega_{R} / \omega}$ & $1.4 \times 10^{-5}\left(\omega_{R} / \omega\right)$ & 0 & $1.0 \times 10^{-13} \sqrt{\omega / \omega_{R}}$ \\
$\left(\omega_{R} / \omega\right) X_{\text {wall }},[\Omega]$ & $4.9 \times 10^{-6} \sqrt{\omega_{R} / \omega}$ & $5.5 \times 10^{-5}$ & $5.5 \times 10^{-6}$ & $3.1 \times 10^{-7}$ \\
\hline \hline
\end{tabular}

external tube, which produces a small resistive component, accounting for radiation leakage through the slots.

We use the following formulas from for thin axial slots from [46]

$$
\begin{aligned}
& \alpha_{\mathrm{tot}}^{(0)}=w^{3}(0.1334-0.005 w / h), \quad \alpha_{\mathrm{tot}}^{(i)}=\left(8 / \pi^{2}\right) \alpha_{\mathrm{tot}}^{(0)}, \\
& \alpha_{\mathrm{tot}}^{(e)}=\exp (-\pi \Delta / w) \alpha_{\mathrm{tot}}^{(0)}
\end{aligned}
$$

where $\alpha_{\mathrm{tot}}^{(0)}$ is the total polarizability of a slot in a vanishingly thin wall, and all other symbols are defined in Fig. 5.

\section{Impedance of OCMF patch}

A straightforward solution, which is not included for brevity, of the electromagnetic boundary value problem for a (relativistic, vanishingly thin) axial beam in a circular OCMF beam screen, with radius $a$ and thickness $\Delta$ surrounded by a coaxial (infinitely thick) conducting circular tube (the cold bore) with radius $b>a+\Delta$ shows that if $\Delta$ exceeds a few skin depths $\delta_{f}$ across the whole beam current spectrum, which is certainly the case here, as seen from Eq. (7), the Leontóvich impedance of the OCMF beam screen wall is fairly well approximated by the intrinsic impedance of the OCMF, viz., [cf. Eq (6)]

$$
Z_{f}=Z_{0}\left(\frac{\nu}{2 \omega_{p}}+\imath \frac{\omega}{\omega_{p}}\right) .
$$

The surface roughness of the foam also contributes to the OCMF wall impedance. The order of magnitude of this contribution can be estimated from [47]

$$
Z_{f}^{(\text {rough })} \approx l \sqrt{\frac{\pi}{32}} Z_{0}\left(\frac{h}{L}\right) \frac{\omega}{c} h,
$$

$h$ and $L$ being the rms height and correlation length of the surface roughness, respectively.

\section{Impedance budget}

For illustrative purposes, the numerical values of the real and imaginary components of the relevant wall impedances, normalized to the mode number [i.e., multiplied by the $\left(\omega_{R} / \omega\right)$ factor], have been collected in Table III. Here we assume high-grade copper $\left(\rho \approx 5.5 \times 10^{-10} \Omega \mathrm{cm}^{-1}\right.$ at $20 \mathrm{~K}$ ) for the solid, slotted, and foam-matrix metal.

The numbers in Table III for the slotted patch are obtained for the slot geometry depicted in Fig. 5, with
$w=\Delta=1.5 \mathrm{~mm}, h=10 \mathrm{w}$, and $s_{T}=s_{L}=2 w$, for which $\xi_{h} \approx 0.14$, and the escape probability reaches its fiducial upper limit $f \approx 0.1$.

The numbers for the foamed patch are obtained assuming a typical pore diameter $\sim 1 \mathrm{~mm}$, and a typical ligament size $\sim 0.1 \mathrm{~mm}$, yielding $\omega_{p} \approx 7.93 \times 10^{10} \mathrm{rad} \mathrm{sec}^{-1}$ and $\nu \approx 3.66 \times 10^{4} \mathrm{~Hz}$ in the Drude model of Sec. II A 1 , consistent with typical measured values of the static conductance of open cell copper foams. We assume a typical rms roughness scale $h \approx 0.125 \mathrm{~mm}$ and a correlation length $L \approx 0.25 \mathrm{~mm}$.

As seen from Table III, the copper wall resistance is larger than that of the OCMF up to $\omega=10^{5} \omega_{R}$. On the other hand, as anticipated in Sec. II A 1, the OCMF wall reactance is relatively large, and exceeds significantly that of a perfectly conducting slotted wall as well as that of solid copper.

However, as seen from Fig. 5, a typical OCMF wall pumping capacity (escape probability) is several times larger than that of the "best" slotted wall.

Thus, to obtain the same pumping capacity, the patched beam screen surface needed in the OCMF case is only a small fraction of that for the slotted case. This makes it easier to place the OCMF patches where the (unperturbed) field in Eq. (16) is minimal, so as to minimize their impact on the beam coupling impedance.

\section{Secondary emission yield}

Perforations reduce the secondary emission yield of a metal wall by a factor roughly proportional to the solid fraction of the surface [6].

The SEY of metals is strongly reduced by surface roughening, obtained, e.g., by powder blasting [8]. For the special case of periodic rectangular grooves etched on a flat metal surface, the SEY dependence on the groove depth-to-spacing and width-to-thickness ratios has been investigated, indicating that larger ratios yield a smaller SEY [48].

Carbon coating also reduces SEY effectively [49], at the expense of some increase in wall resistance.

It is reasonable to expect that OCMFs may exhibit a much lower SEY compared to solid metals in view of their randomlike surface roughness. The SEY of an OCMF wall will depend on the effective roughness of its surface, which is a function of the foam porosity and average pore size. OCMF surface roughness, however, should be kept small enough, to prevent the blowup of 
wall reactance. No measurements of the SEY of OCMFs are available yet, to the best of our knowledge, but should be straightforward [50].

\section{CONCLUSIONS}

On the basis of the above discussion, some tentative conclusions can be drawn about the possible use of OCMF materials in the beam screens of high synchrotron radiation accelerators.

The outgassing capabilities of OCMF walls can be better by a factor $\sim 10$ compared to the best slotted walls, and would accordingly allow one to boost the molecular escape probability of beam screens by roughly one order of magnitude, compared to present designs, while keeping the permeable fraction of the beam screen surface unchanged.

The mechanical-structural properties of conducting foams should also be adequate to resist eddy current induced stresses, in view of their morphology. A low SEY is also expected, due to OCMF surface roughness, even though no experimental results are available yet.

The OCMF surface resistance is very low, and almost frequency independent. On the other hand, the (inductive) wall reactance of an OCMF patch is larger by roughly one order of magnitude compared to that of a perforated wall with comparable escape probability. This drawback could be mitigated by clever placement of the OCMF patches, and/or appropriate reactive loading of the (solid portion of) the pipe wall.

In order to translate the above points into effective design criteria, further modeling effort and substantial experimental work are in order. Measurement of the complex surface impedance and SEY of metal foams are now underway [51].

We believe that such an effort is worth being pursued, and that the available modeling tools and technologies provide a good starting point for its successful implementation.

\section{ACKNOWLEDGMENTS}

This work has been sponsored in part by the Italian Institute for Nuclear Physics (INFN) under the IMCA grant. Stimulating discussions with F. Zimmermann and G. Rumolo (CERN) are gratefully acknowledged.

[1] A. Valishev, arXiv:1108.1644v1.

[2] A. Blondel et al., arXiv:1208.0504v2.

[3] CERN, Large Hadron Collider Conceptual Design Report No. AC/95-05, 1995 [http://cds.cern.ch/record/291782/ files/cm-p00047618.pdf].

[4] B. W. Zotter and S. A. Kheifets, Impedances and Wakes in High Energy Particle Accelerators (World Scientific, Singapore, 1998).

[5] S. Heifets and A. Mikhailichenko, SLAC Pub. No. 8428, 2000 [http://slac.stanford.edu/cgi-wrap/getdoc/slac-pub8428.pdf].
[6] I. Montero, L. Aguilera, L. Galán, V. Nistor, J. L. Sacedón, M.Vázquez, F. Caspers, D. Raboso et al., in Proceedings of AEC 'O9 (CERN, Geneva, Switzerland, 2009) [https:// indico.cern.ch/event/62873/session/4/contribution/29].

[7] F. Zimmermann, Phys. Rev. ST Accel. Beams 7, 124801 (2004).

[8] K. C. Harkay, in Proceedings of the EPAC '06, Edinburgh, Scotland, 2006(EPS-AG, Edinburgh, Scotland, 2006) [http:// accelconf.web.cern.ch/AccelConf/e06/PAPERS/WEXFI02 .PDF].

[9] J. Banhart and N. A. Fleck, Cellular Metals and Metal Foaming Technology (MIT Press, Boston, 2003).

[10] Erg Aerospace Corporation, www.ergaerospace.com.

[11] D. Weaire and R. Phelan, Philos. Mag. Lett. 69, 107 (1994).

[12] 3D morphological software for porous media analysis, www.imorph.sourceforge.net.

[13] T. Weiland, Electron. Commun. (AEU) 31, 116 (1977).

[14] H. Zhang, J. Zhang, and H. Zhang, Smart Mater. Struct. 15, 759 (2006).

[15] F. G. Cuevas, J. M. Montes, J. Cintas, and P. Urban, J. Porous Mater. 16, 675 (2009).

[16] R. Goodall, L. Weber, and A. Mortensen, J. Appl. Phys. 100, 044912 (2006).

[17] D. A. G. Bruggemann, Ann. Phys. (N.Y.) 24, 636 (1935).

[18] R. McLaughlin, Int. J. Eng. Sci. 15, 237 (1977).

[19] Y. Qui and G. J. Weng, Int. J. Eng. Sci. 28, 1121 (1990).

[20] S. K. Kanaun, Int. J. Eng. Sci. 41, 1287 (2003).

[21] T.W. Clyne, in Comprehensive Composite Materials (Elsevier, Amsterdam, 2000).

[22] D. Stauffer and A. Aharony, Introduction to percolation Theory (Taylor and Francis, London, 1992).

[23] I. Sevostianov, J. Kovacic, and F. Simancik, Mater. Sci. Eng. A 420, 87 (2006).

[24] G. Monti, L. Catarinucci, and L. Tarricone, Microwave Opt. Technol. Lett. B52, 1700 (2010).

[25] J. B. Pendry, A. J. Holden, D. J. Robbins, and W. J. Stewart, J. Phys. Condens. Matter 10, 4785 (1998).

[26] M. A. Ordal, L. L. Long, R. J. Bell, S. E. Bell, R. R. Bell, R. W. Alexander, Jr., and C. A. Ward, Appl. Opt. 22, 1099 (1983).

[27] H. Day, F. Caspers, A. Dallocchio, L. Gentini, A. Grudiev, E. Metral, B. Salvant, and R. M. Jones, Proceedings of IPAC'12, New Orleans, LA, 2012 (IEEE, Piscataway, NJ, 2012) [http://accelconf.web.cern.ch/accelconf/ipac2012/ papers/weppr070.pdf].

[28] E. S. Reddy and G. J. Schmitz, Supercond. Sci. Technol. 15, L21 (2002).

[29] E. S. Reddy, M. Herweg, and G. J. Schmitz, Supercond. Sci. Technol. 16, 608 (2003).

[30] E. Bartolomé, X. Granados, T. Puig, X. Obradors, E. Reddy, and G. Schmitz, Phys. Rev. B 70, 144514 (2004).

[31] D. N. Basov et al., Rev. Mod. Phys. 77, 721 (2005).

[32] C. A. M. dos Santos, C. Oliveira, M. da Luz, A. Bortolozo, M. Sandim, and A. Machado, Phys. Rev. B 74, 184526 (2006).

[33] J. H. Ma and I. Wolf, IEEE Trans. Microwave Theory Tech. 43, 1053 (1985).

[34] J. G. Noudem, Physica C (Amsterdam) 390, 286 (2003).

[35] O. Gröbner, Vacuum 60, 25 (2001).

[36] W. Steckelmacher, Rep. Prog. Phys. 49, 1083 (1986). 
[37] O Malyshev, CERN, LHC Vacuum Technical Note 01-06, 2001 [http://at-div-vac.web.cern.ch/at-div-vac/VACPAGES/ VacuumTechNote/2001/TN_01_06.pdf].

[38] W. Steckelmacher and K. F. Man, Rev. Sci. Instrum. 56, 58 (1985).

[39] T. Fiedler, I. V. Belova, and G. E. Murch, Comput. Mater. Sci. 50, 1795 (2011).

[40] T. Fiedler, I. V. Belova, and G. E. Murch, Comput. Mater. Sci. 50, 2666 (2011).

[41] S. Petracca, Part. Accel. 50, 211 (1995).

[42] A. M. Dykne and I. M. Kaganova, Opt. Commun. 206, 39 (2002).

[43] S. S. Kurennoy, Part. Accel. 39, 1 (1992).

[44] S. Petracca, Phys. Rev. E 60, 6030 (1999).

[45] R. L. Gluckstern and B. W. Zotter, CERN Report No. SL 96/56 (AP), 1996 [http://cds.cern.ch/record/309563/files/ sl-96-056.pdf].
[46] S. Kurennoy, KEK-preprint No. 94-193, 2005 [http:// inspirehep.net/record/393006? ln=en].

[47] K. L. F. Bane and G. V. Stupakov, SLAC Pub. 8023, 1988 [http://www.slac.stanford.edu/cgi-wrap/getdoc/slacpub-8023.pdf].

[48] M. Pivi et al., SLAC Pub. 13020, 2007 [http://www.slac .stanford.edu/cgi-wrap/getdoc/slac-pub-13020.pdf].

[49] C. Yin Vallgren et al., Phys. Rev. ST Accel. Beams 14, 071001 (2011).

[50] F. Caspers et al., in Proceedings of EPAC '02, Paris (EPS-IGA and CERN, Geneva, 2002), WEPDO031 [https://accelconf.web.cern.ch/accelconf/e02/PAPERS/ WEPDO031.pdf].

[51] R. Cimino, A. Romano, S. Petracca, A. Stabile, M. R. Masullo, S. O'Connor, G Bregliozzi, V. Baglin, in Proceedings of IPAC '14, Dresden, German, 2013, WEPME033 [http://accelconf.web.cern.ch/AccelConf/IPAC2014/papers/ wepme033.pdf]. 